\title{
Identification of the bulk pairing symmetry in high-temperature superconductors: Evidence for an extended s-wave with eight line nodes
}

\author{
Guo-meng Zhao \\ Physik-Institut der Universität Zürich, CH-8057 Zürich, Switzerland
}

\begin{abstract}
we identify the intrinsic bulk pairing symmetry for both electron and hole-doped cuprates from the existing bulk- and nearly bulk-sensitive experimental results such as magnetic penetration depth, Raman scattering, single-particle tunneling, Andreev reflection, nonlinear Meissner effect, neutron scattering, thermal conductivity, specific heat, and angle-resolved photoemission spectroscopy. These experiments consistently show that the dominant bulk pairing symmetry in hole-doped cuprates is of extended $s$-wave with eight line nodes, and of anisotropic $s$-wave in electron-doped cuprates. The proposed pairing symmetries do not contradict some surface- and phase-sensitive experiments which show a predominant $d$-wave pairing symmetry at the degraded surfaces. We also quantitatively explain the phase-sensitive experiments along the c-axis for both $\mathrm{Bi}_{2} \mathrm{Sr}_{2} \mathrm{CaCu}_{2} \mathrm{O}_{8+y}$ and $\mathrm{YBa}_{2} \mathrm{Cu}_{3} \mathrm{O}_{7-y}$.
\end{abstract}

\section{INTRODUCTION}

An unambiguous determination of the symmetry of the order parameter (pair wavefunction) in cuprates is crucial to the understanding of the pairing mechanism of high-temperature superconductivity. In recent years, many experiments have been designed to test the order parameter (OP) symmetry in the cuprate superconductors. However, contradictory conclusions have been drawn from different experimental techniques [1] [16], which can be classified into being bulk-sensitive and surface-sensitive. For example, the magnetic penetration depth measurements and polarized Raman scattering experiments are bulk-sensitive. Angle-resolved photoemission spectroscopy (ARPES) is essentially a surface-sensitive technique. However, the ARPES data for $\mathrm{Bi}_{2} \mathrm{Sr}_{2} \mathrm{CaCu}_{2} \mathrm{O}_{8+y}$ (BSCCO) should nearly reflect the bulk properties since the cleaved top surface contains an inactive Bi-O layer, and the superconducting coherent length along the c-axis is very short. The singleparticle tunneling experiments can probe the bulk electronic density of states when the mean free path is far larger than the thickness of the degraded surface layer [17]. Therefore, the single-particle tunneling experiments along the $\mathrm{CuO}_{2}$ planes are almost bulk-sensitive due to a large in-plane mean free path $(>100 \AA)$. In contrast, the phase-sensitive experiments based on the Josephson tunneling are rather surface sensitive (since pair tunneling is limited by the coherence length which is rather short in cuprates), so that they might not probe the intrinsic bulk superconducting state if the surfaces are strongly degraded. In this case, the observed product of the critical current times the junction normal-state resistance $\left(I_{c} R_{N}\right)$ will be very small compared with the Ambegaokar-Baratoff limit. Then the OP symmetry at surfaces may be different from the one in the bulk [18]. Therefore, the surface- and phase-sensitive experiments do not necessarily provide an acid test for the intrinsic bulk OP symmetry.
Here, we identify the intrinsic bulk pairing symmetry for both electron and hole-doped cuprates from the existing bulk- and nearly bulk-sensitive experimental results such as magnetic penetration depth, Raman scattering, single-particle tunneling, Andreev reflection, nonlinear Meissner effect, neutron scattering, thermal conductivity, specific heat, and ARPES. These experiments consistently show that the dominant bulk pairing symmetry in hole-doped cuprates is of extended $s$-wave with eight line nodes, and of anisotropic $s$-wave in electrondoped cuprates. The proposed pairing symmetries do not contradict some surface- and phase-sensitive experiments which show a $d$-wave pairing symmetry at the degraded surfaces. The extended $s$-wave pairing symmetry deduced from the bulk-sensitive experiments is also in quantitative agreeement with the well-designed phase-sensitive experiments along the c-axis for both $\mathrm{Bi}_{2} \mathrm{Sr}_{2} \mathrm{CaCu}_{2} \mathrm{O}_{8+y}$ and $\mathrm{YBa}_{2} \mathrm{Cu}_{3} \mathrm{O}_{7-y}$.

\section{THE PAIRING SYMMETRY IN HOLE-DOPED CUPRATES}

\section{A. The pairing symmetry in $\mathrm{Bi}_{2} \mathrm{Sr}_{2} \mathrm{CaCu}_{2} \mathrm{O}_{8+y}$}

We first examine the high-resolution ARPES data obtained for $\mathrm{Bi}_{2} \mathrm{Sr}_{2} \mathrm{CaCu}_{2} \mathrm{O}_{8+y}$ crystals [14 16]. From the ARPES data, one can determine the angle dependence of the superconducting gap with a resolution as high as \pm 2 $\mathrm{meV}$ [16]. Due to the complication arising from a possible superlattice contribution in the $\mathrm{X}$ quadrant, we only use the data obtained for the Y quadrant to extract the gap function. In Fig. 1, we show the angle dependence of the superconducting gap $\Delta(\theta)$ in the Y quadrant for slightly overdoped and heavily overdoped BSCCO single crystals. The data were taken from Ref. 14, 16]. Here $\theta$ is the angle measured from the $\mathrm{Cu}-\mathrm{O}$ bonding direction. For the slightly overdoped sample (Fig. 17a), the gap $\Delta_{D}$ at $\theta$ $=45^{\circ}$ (diagonal direction) is very small $(3.5 \pm 2.5 \mathrm{meV})$, 
and the gap symmetry could be consistent with a $d$-wave symmetry, i.e., $\Delta(\theta)=\Delta \cos 2 \theta$. On the other hand, the gap along the diagonal direction $(\Gamma-Y)$ for the heavily overdoped sample (Fig. 1 $\mathrm{b}$ ) is not small $(9 \pm 2 \mathrm{meV})$, which is obviously not consistent with the $d$-wave pairing symmetry. A similar evolution of the gap function with the doping has been observed by the bulk-sensitive polarized Raman scattering [7], which also shows that the difference in the magnitudes of the gaps along the $\mathrm{Cu}-\mathrm{O}$ bonding direction and the diagonals becomes smaller and smaller towards overdoping.

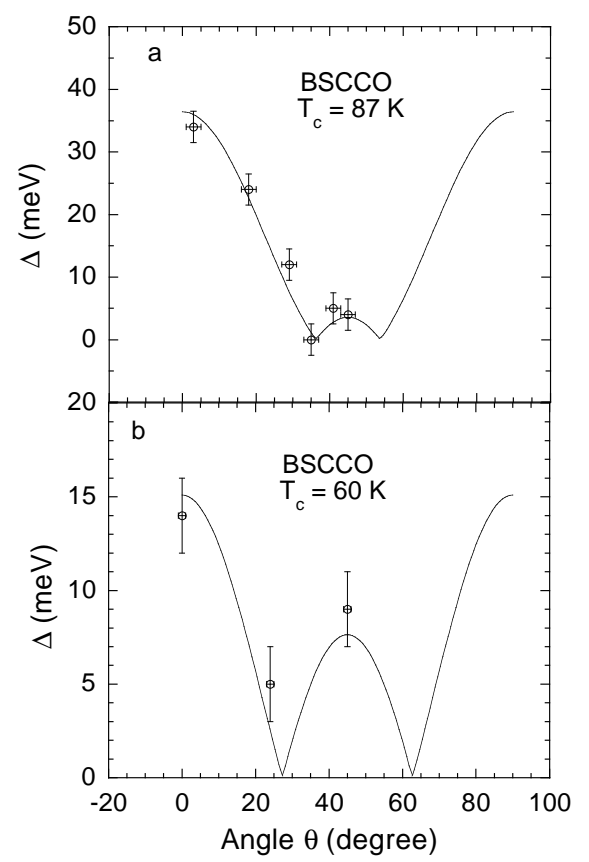

FIG. 1. The angle dependence of the superconducting gap $\Delta(\theta)$ in the $\mathrm{Y}$ quadrant for $\mathrm{Bi}_{2} \mathrm{Sr}_{2} \mathrm{CaCu}_{2} \mathrm{O}_{8+y}$ (BSCCO) crystals: (a) slightly overdoped sample with $T_{c}=87 \mathrm{~K}$; (b) heavily overdoped sample with $T_{c}=60 \mathrm{~K}$. The magnitudes of the gap were extracted from ARPES data 14, 16. Here $\theta$ is the angle measured from the $\mathrm{Cu}-\mathrm{O}$ bonding direction.

The question is: what functional form of $\Delta(\theta)$ can fit the angle dependence of the gap shown in Fig. 11? In general, the gap can be expressed as $\Delta(\theta)=\Delta_{s}+$ $\Delta_{d} \cos 2 \theta+\Delta_{g} \cos 4 \theta+\ldots$ In the case of $\Delta_{d} \simeq 0$, one has

$$
\Delta(\theta)=\Delta(\cos 4 \theta+s)
$$

where $s$ is the parameter reflecting the isotropic $s$-wave component. This gap function has eight line nodes for $s<1$, while there are no nodes for $s>1$. The gap function (Eq. 1) is also called extended $s$-wave (denoted by $s^{*}$-wave). The polarized Raman data for an optimally doped $\mathrm{HgBa}_{2} \mathrm{CaCu}_{2} \mathrm{O}_{6+y}$ are in good agreement with the $s^{*}$-wave gap function [6]. If we take the absolute value of
$\Delta(\theta)$, then

$$
|\Delta(\theta)|=|\Delta(\cos 4 \theta+s)| .
$$

We fit the data of Fig. 1 by Eq. 2. It is remarkable that the fits are rather good. This indicates that the ARPES data may be consistent with the extended $s$-wave symmetry. The ARPES specified maximum gap $\Delta_{M}$ at $\theta=$ 0 for the slightly overdoped sample is $36 \pm 3 \mathrm{meV}$, which is much larger than the value $(\sim 28 \mathrm{meV})$ determined from break junction spectra [21]. On the other hand, the ARPES determined $\Delta_{M}$ value $(15 \pm 2 \mathrm{meV})$ for the heavily overdoped sample with $T_{c}=60 \mathrm{~K}$ is very close to the value $(18 \pm 2 \mathrm{meV})$ inferred from a break junction spectrum of a similar crystal with $T_{c}=62 \mathrm{~K}$ [22]. The discrepancy in the former case may be due to the fact that the doping level in the top layer where the ARPES probes could be slightly lower than in the bulk (i.e., the top $\mathrm{CuO}_{2}$ layer might be slightly underdoped). Thus, the ARPES experiments on the BSCCO single crystals are nearly bulk sensitive, in contrast to the ARPES experiments on other cuprates, which are essentially surface sensitive.

If the proposed gap functions (Eq. 1] and Eq. 2) are indeed relevant, they should be also consistent with other bulk-sensitive experimental results such as the in-plane magnetic penetration depth $\lambda_{a b}(T)$. Since there are eight line nodes in the proposed gap function, the change of the in-plane penetration depth at low temperatures should be proportional to $T$. Following the procedure in Ref. [23], we can readily show that the slope

$$
d \lambda_{a b}(T) / d T=\left[\lambda_{a b}(0) \ln 2 / \Delta_{M}\right] \sqrt{(1+s) /(1-s)} .
$$

Compared with the $d$-wave symmetry, the magnitude of the slope $d \lambda_{a b}(T) / d T$ is enhanced by a factor of $\sqrt{(1+s) /(1-s)}$. In terms of $\Delta_{M}$ and $\Delta_{D}$, we find that $s=\left(\Delta_{M}-\Delta_{D}\right) /\left(\Delta_{M}+\Delta_{D}\right)$ and $\Delta=\left(\Delta_{M}+\Delta_{D}\right) / 2$. Then, Eq. 3 can be rewritten as

$$
d \lambda_{a b}(T) / d T=\lambda_{a b}(0) \ln 2 / \sqrt{\Delta_{M} \Delta_{D}} .
$$

It is interesting to see that $d \lambda_{a b}(T) /\left[\lambda_{a b}(0) d T\right]$ is inversely proportional to $\sqrt{\Delta_{M} \Delta_{D}}$, namely, the geometric average of $\Delta_{M}$ and $\Delta_{D}$.

The single-particle tunneling spectroscopy can probe the superconducting density of states (DOS) with fine energy resolution and considerable directionality. For an isotropic $s$-wave superconductor, the characteristic $d I / d V$ vs $V$ curve in the point-contact SIN tunneling junctions exhibits a step-like peak at a voltage $V_{p}=\Delta / e$. For an anisotropic gap function $\Delta(\theta)$, the directional dependence of the tunneling differential conduction is given by 24.

$$
\frac{d I}{d V} \propto \int_{0}^{2 \pi} p\left(\theta-\theta_{0}\right) \Re\left[\frac{e V-i \Gamma}{\sqrt{(e V-i \Gamma)^{2}-\Delta^{2}(\theta)}}\right] N(\theta) d \theta .
$$


Here $N(\theta)$ represents the anisotropy of the band dispersion; $\Gamma$ is the life-time broadening parameter of an electron; $p\left(\theta-\theta_{0}\right)$ is the angle dependence of the tunneling probability which decays exponentially as $p\left(\theta-\theta_{0}\right)=$ $\exp \left[-\beta \sin ^{2}\left(\theta-\theta_{0}\right)\right]\left(\theta_{0}\right.$ is the angle of the tunneling barrier direction); the parameter $\beta$ decreases with decreasing barrier resistance $R_{N}$. For simplicity, we assume a cylindrical Fermi surface, so that both $N(\theta)$ and $\beta$ are independent of the angle. This will not change the basic features of the $d I / d V$ curve. In Fig. 2 we show the numerically calculated results of the renormalized $d I / d V$ for a gap function of $\Delta(\theta)=\Delta(\cos 4 \theta+s)$ with $\Delta=24$ $\mathrm{meV}$ and $s=0.25$. One can readily show that the maximum gap is $\Delta_{M}=(1+s) \Delta=30 \mathrm{meV}$ at $\theta=0$, and the gap along the diagonal directions is $\Delta_{D}=(1-s) \Delta$ $=18 \mathrm{meV}$. From Fig. 2, one can see that either two or four peak features appear clearly in the $d I / d V$ curves, depending on the tunneling barrier direction and/or the $\beta$ value. For a small $\beta$ value (corresponding to a small barrier resistance), four peak features are well defined (see curve A). The peak positions are located at $e V=$ $\pm \Delta_{M}$ and $\pm \Delta_{D}$. Therefore, from the peak positions, we can determine $\Delta_{M}$ and $\Delta_{D}$.

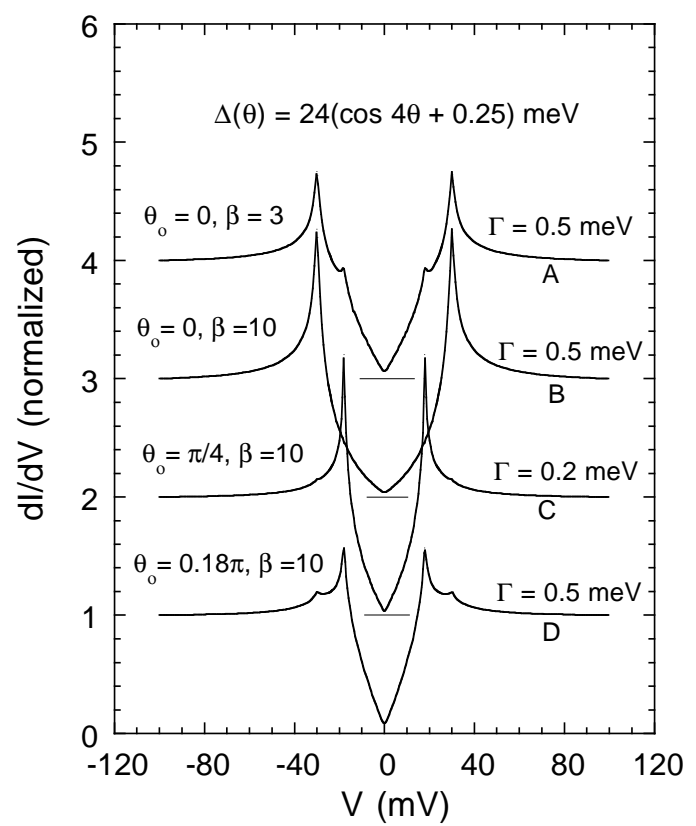

FIG. 2. Numerically calculated curves of the renormalized $d I / d V$ for a gap function of $\Delta(\theta)=\Delta(\cos 4 \theta+s)$ with $\Delta=$ $24 \mathrm{meV}$ and $s=0.25$. The four curves correspond to different values of the parameters $\Gamma, \beta$, and $\theta_{o}$, which are indicated in the figure. The curves $\mathrm{A}, \mathrm{B}$ and $\mathrm{C}$ are vertically shifted up by 3,2 and 1 , respectively.

In Fig. 3, we plot the normalized $d I / d V$ curve at 14 $\mathrm{K}$ for an SIS break junction on a BSCCO crystal which is slightly overdoped $\left(T_{c}=90 \mathrm{~K}\right)$ 25]. The junction has a very low barrier resistance $(\sim 200 \Omega)$ [25], indicating a small $\beta$ value. It is remarkable that there are four well-defined peak features in the spectrum, which resemble curve A in Fig. 2. The pronounced zero bais peak arises from Josephson tunneling [21.25]. When the barrier resistance is above $2 \mathrm{k} \Omega$, the inner gap features disappear [25], in agreement with curve B in Fig. 2. We would like to mention that, for SIS break junctions, the peak positions are located at $e V= \pm 2 \Delta_{M}$ and $\pm 2 \Delta_{D}$. From the spectra, we obtain $\Delta_{M}=26 \pm 0.5 \mathrm{meV}$, and $\Delta_{D}=9.5 \pm 0.5 \mathrm{meV}$. The $\Delta_{M}$ value obtained from the break junction spectrum is the same as that found from the c-axis intrinsic tunneling junctions made of the insulating Bi-O layers [26]. From the $\Delta_{M}$ and $\Delta_{D}$ values, we deduce a gap function $\Delta(\theta)=\Delta(\cos 4 \theta+s)$ with $\Delta=17.75 \mathrm{meV}$ and $s=0.46$. With this gap function and $\lambda_{a b}(0)=2690 \pm 150 \AA$ [27], we calculate from Eq. 4 that $d \lambda_{a b}(T) / d T=10.2 \pm 0.6 \AA / \mathrm{K}$, in excellent agreement with the measured values $(10.2 \pm 0.2 \AA / \mathrm{K})$ [2,3]. Similarly, the earlier break junction spectra for an overdoped BSCCO with $T_{c}=86 \mathrm{~K}$ also indicate double gap features at $\Delta_{M}=24 \pm 2 \mathrm{meV}$ and at $\Delta_{D}=12 \pm 1 \mathrm{meV}$ (Ref. [28]). The tunneling spectra are in good agreement with ARPES data for an overdoped BSCCO with $T_{c}=$ $83 \mathrm{~K}$ [19]. The ARPES experiment clearly showed that $\Delta_{M}=20 \pm 2 \mathrm{meV}$ and $\Delta_{D}=12 \pm 2 \mathrm{meV}$ [19]. Moreover, the inner gap features also appear in SIS break junction spectra of a heavily overdoped crystal with $T_{c}=$ $62 \mathrm{~K}$, corresponding to $\Delta_{D}=7.5-9.0 \mathrm{meV}$ (Ref. [22, 29]). The magnitude of $\Delta_{D}$ is in excellent agreement with that found from the ARPES experiment (see Fig. 10b).

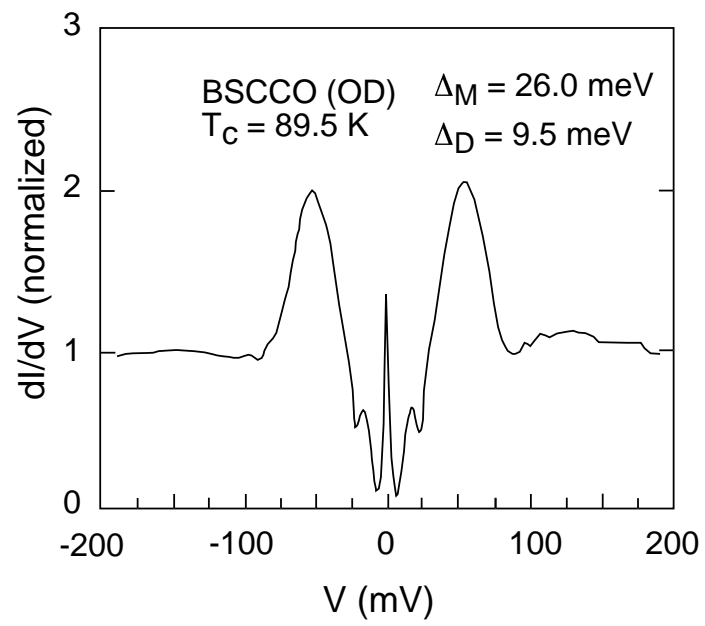

FIG. 3. Normalized $d I / d V$ curves at $14 \mathrm{~K}$ for the SIS break junctions on a slightly overdoped BSCCO crystal. The spectra were taken from Ref. [25].

We would like to point out that the values of $\Delta_{M}$ determined from Raman spectrum of $B_{1 g}$ symmetry may be overestimated due to the fact that the extended van Hove singularity is slightly below the Fermi level. In this case, the spectra would show double peaks at Raman shifts of $2 \Delta_{M}$ and $2 \sqrt{\Delta_{M}^{2}+\xi_{v H}^{2}}$, where $\xi_{v H}$ is the energy posi- 
tion of the van Hove singularity below the Fermi level. When $\xi_{v H}<<\Delta_{M}$, one can only see a single broad peak slightly below $2 \sqrt{\Delta_{M}^{2}+\xi_{v H}^{2}}$.

\section{B. The pairing symmetry in $\mathrm{YBa}_{2} \mathrm{Cu}_{3} \mathrm{O}_{7-y}$}

Evidence for an extended $s$-wave pairing symmetry in $\mathrm{YBa}_{2} \mathrm{Cu}_{3} \mathrm{O}_{7-y}$ (YBCO) also comes from single-particle tunneling spectra. Fig. 4 shows scanning tunneling spectrum for a slightly overdoped $\mathrm{YBa}_{2} \mathrm{Cu}_{3} \mathrm{O}_{7-y}$ crystal [30]. Four peak features appear in this spectrum that is similar to curve D in Fig. 2. From the peak positions, we obtain $\Delta_{M}=30 \pm 2 \mathrm{meV}$, and $\Delta_{D}=19 \pm 1 \mathrm{meV}$. The size of $\Delta_{M} \simeq 30 \mathrm{meV}$ is consistent with a break junction spectrum 17, and a scanning tunneling spectrum along the a-axis direction [8]. A gap feature with $\Delta_{D}=19$ meV was also seen in a scanning tunneling spectrum \& that is very similar to curve $\mathrm{C}$ in Fig. 2.

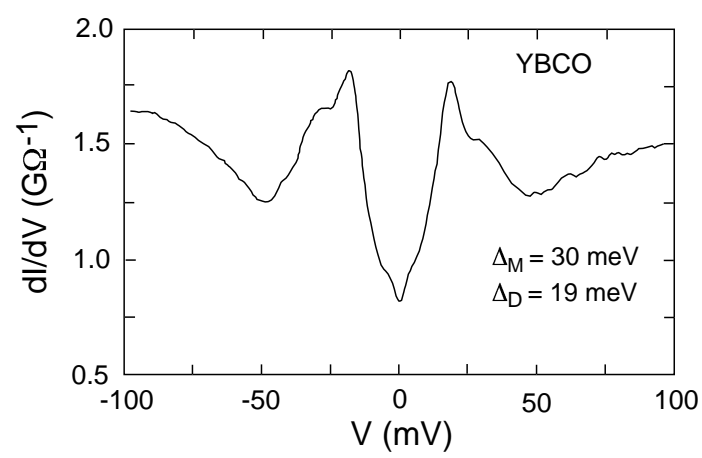

FIG. 4. Scanning tunneling spectrum for a slightly overdoped $\mathrm{YBa}_{2} \mathrm{Cu}_{3} \mathrm{O}_{7}$ (YBCO) crystal. The spectrum was taken from Ref. [30].

Now we discuss the Andreev reflection. Since there is sign change about its nodal directions in our extended $s$ wave order parameter, the Andreev-bound surface states can be formed. This will lead to a zero-bias conduction peak (ZBCP) if tunneling is nearly along one of the nodal directions and the bare Fermi velocities between the cuprates and normal metals (e.g., Ag and Au) are well matched. For hole doped cuprates, the bare Fermi velocity $v_{F}^{b}$ strongly depend on the angle $\theta$, that is, $v_{F}^{b}$ is small along the bonding direction, and large along the diagonal directions. This implies that the observation of the Andreev reflection is difficult for tunneling along the bonding direction since the value of $v_{F}^{b}$ along this direction is small compared with that of $\mathrm{Au}$ or $\mathrm{Ag}$. Due to the strong anisotropy of $v_{F}^{b}$ in cuprates, the Andreev reflection mainly probes the gap feature at $e V=\Delta_{D}$. If tunneling is along one of the diagonal directions, and the angle between the nodel and diagonal directions is far larger than the half tunneling angle (depending on $\beta$ ), one can see an $s$-wave like gap approximately equal to
$\Delta_{D}$ in the Andreev reflection spectra. Indeed an $s$-wave like gap feature at $e V \simeq 20 \mathrm{meV}$ has been observed in the Andreev reflection spectra of several YBCO crystals with $T_{c}=90 \mathrm{~K}$ 31. We would like to mention that, in general, the double gap features should also appear in the Andreev reflection spectra when the $\beta$ value is small and $v_{F}^{b}$ does not have a significant anisotropy.

The tunneling data of YBCO (Fig. 4) are thus consistent with a gap function $\Delta(\theta)=\Delta(\cos 4 \theta+s)$ with $\Delta=$ $24.5 \mathrm{meV}$ and $s=0.225$. This gap function is in quantitative agreement with the a-axis $\lambda_{a}(T)$ data (which reflect magnetic screening in $\mathrm{CuO}_{2}$ planes) for a fully oxygenated YBCO crystal [32]. From Eq. A, we calculate $d \lambda_{a}(T) / d T=4.0 \AA / \mathrm{K}$ using $\lambda_{a}(0)=1600 \AA$ (Ref. [32]), $\Delta_{D}=19 \mathrm{meV}$, and $\Delta_{M}=30 \mathrm{meV}$. We will get the same value of $d \lambda_{a}(T) / d T$ if we use $\Delta_{D}=21 \mathrm{meV}$, and $\Delta_{M}$ $=27 \mathrm{meV}$. For a $d$-wave gap function $\Delta(\theta)=\Delta_{M} \cos 2 \theta$ with $\Delta_{M}=30 \mathrm{meV}$, the calculated $d \lambda_{a}(T) / d T=3.2$ $\AA / \mathrm{K}$. The measured value of $d \lambda_{a}(T) / d T$ is $4 \AA / \mathrm{K}[32$. It is evident that the extended $s$-wave gap function is in much better agreement with experiment than the $d$-wave gap function.

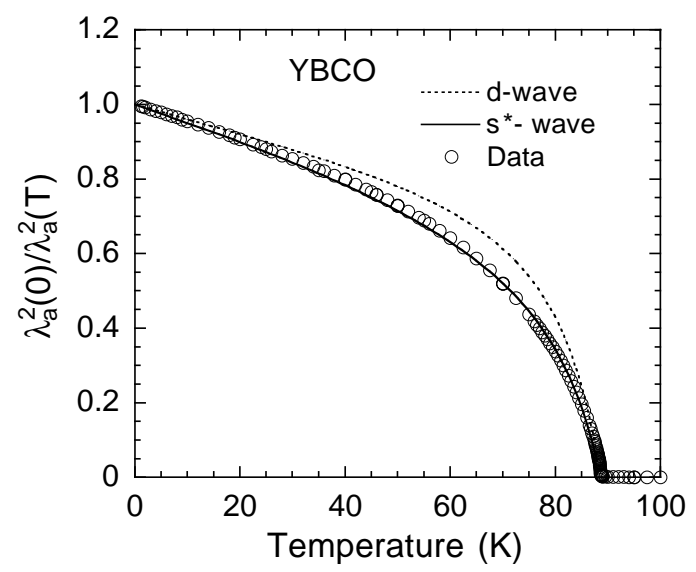

FIG. 5. Temperature dependence of the a-axis $\lambda_{a}^{2}(0) / \lambda_{a}^{2}(T)$ for a very high-quality $\mathrm{YBa}_{2} \mathrm{Cu}_{3} \mathrm{O}_{7}$ (YBCO) crystal with $T_{c}$ $=88.7 \mathrm{~K}$. The data were taken from Ref. 32. The solid line is the calculated curve for the $s^{*}$-wave gap function deduced from the tunneling spectrum in Fig. 4. The dash line is the calculated curve for a $d$-wave gap function with $\Delta_{M}=30$ $\mathrm{meV}$.

Now we calculate the temperature dependence of $\lambda_{a b}^{2}(0) / \lambda_{a b}^{2}(T)$ for the $s^{*}$-wave gap function. For a cylindrical Fermi surface [2]

$$
\frac{\lambda_{a b}^{2}(0)}{\lambda_{a b}^{2}(T)}=1+(1 / \pi) \int_{0}^{2 \pi} \int_{0}^{\infty} d \theta d \epsilon \frac{\partial f}{\partial E} .
$$

Here $E=\sqrt{\epsilon^{2}+\Delta^{2}(\theta, T)} ; f$ is the Fermi-Dirac distribution function; $\Delta(\theta, T)=\Delta(T)(\cos 4 \theta+s) ; \Delta(T)=$ $\Delta \tanh \left(2.2 \sqrt{T / T_{c}-1}\right)$ (Ref. [33]). In Fig. 5, we compare the experimental data for YBCO (open circles) 32] 
and the numerically calculated result (solid line) for the above deduced gap function $\Delta(\theta)=24.5(\cos 4 \theta+0.225)$ $\mathrm{meV}$. It is remarkable that the data are in quantitative agreement with the calculated result without any fitting parameters. The dash line is the calculated result for a $d$-wave gap function $\Delta(\theta)=\Delta_{M} \cos 2 \theta$ with $\Delta_{M}=30$ $\mathrm{meV}$. It is clear that the agreement between the data and the calculated curve is poor for the $d$-wave symmetry. It is worthy to note that the temperature dependence of $\lambda_{a b}^{2}(0) / \lambda_{a b}^{2}(T)$ is mainly determined by the gap function, so the shape of the Fermi surface has little effect on $\lambda_{a b}^{2}(0) / \lambda_{a b}^{2}(T)$.

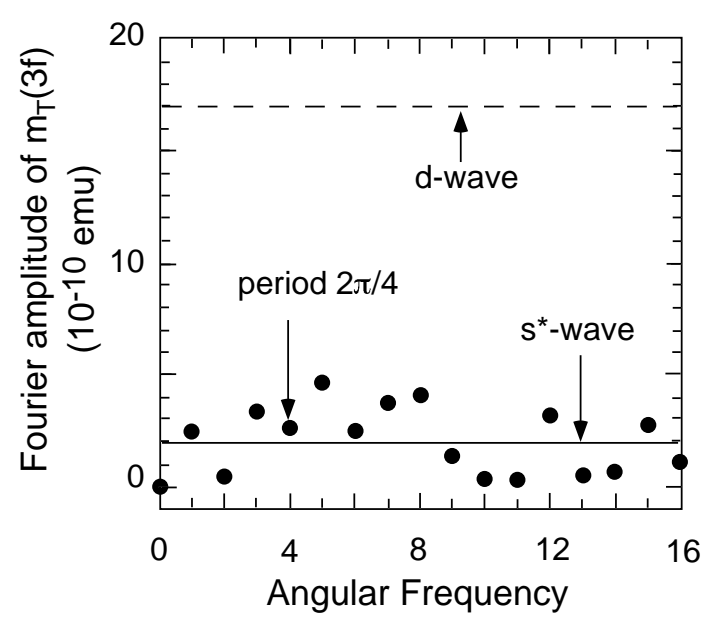

FIG. 6. Sine Fourier amplitudes of the transverse magnetization $m_{T}$ in the Meissner state for a high-quality $\mathrm{YBa}_{2} \mathrm{Cu}_{3} \mathrm{O}_{7}$ (YBCO) crystal. The data were taken from Ref. [4]. The solid line is the predicted sine Fourier amplitude at $2 \pi / 4$ for the $s^{*}$-wave gap function deduced from the tunneling spectrum in Fig. 1 and the a-axis $\lambda_{a}(T)$ data in Fig. 5 . The calculated amplitudes at $2 \pi / 2,2 \pi / 3,2 \pi / 4$, and $2 \pi / 5$ are similar while the ones at other periods are much smaller. The dash line is the predicted sine Fourier amplitude at $2 \pi / 4$ for a $d$-wave gap function.

The gap function of YBCO deduced from the tunneling and the $\lambda_{a}(T)$ data is also consistent with the measured transverse magnetization $m_{T}$ in the Meissner state [4], as plotted in Fig. 6. This bulk sensitive experiment shows a very small sine fourfold component of the transverse magnetization, which is at least 4 times smaller than the predicted value from the $d$-wave symmetry. This indicates that the dominant pairing symmetry is not the $d$ wave. Using the formulas reported in Ref. [34], we can calculate the sine components of the transverse magnetization for the $s^{*}$-wave gap function deduced above. We find that the sine fourfold component for the $s^{*}$-wave OP is a factor of 8.9 smaller than for the pure $d$-wave OP. The predicted sine Fourier amplitude at period $2 \pi / 4$ is indicated by a horizontal solid line in Fig. 6. The calculated amplitudes at $2 \pi / 2,2 \pi / 3,2 \pi / 4$, and $2 \pi / 5$ are similar while the ones at other periods are much smaller.
It is clear that the predicted amplitudes at all the periods are below the noise level which is about $5 \times 10^{-10}$ emu [4]. Therefore, the very small nonlinear Meissner effect observed in the overdoped YBCO is in agreement with the $s^{*}$-wave OP or with a nodeless OP [4] rather than with the $d$-wave OP. A nodeless OP symmetry is in contradiction with the observed linear $T$ dependence of the thermal conductivity down to a very low temperature (50 mK) 35.

In addition, we further show that the $s^{*}$-wave gap function is in quantitative agreement with the low temperature thermal conductivity, specific heat, and surface Andreev bound states. By replacing $\Delta_{M}$ by $\sqrt{\Delta_{M} \Delta_{D}}$ in the equations for the low-temperature electronic thermal conductivity $\kappa_{e l}$ and specific heat $C_{e l}$ for the $d$-wave gap function in the clean limit [35], we obtain the following equations for an $s^{*}$-wave gap function:

$$
\frac{\kappa_{e l}}{T}=\frac{k_{B}^{2} v_{F} k_{F}}{6 d \sqrt{\Delta_{M} \Delta_{D}}},
$$

and

$$
\frac{C_{e l}}{T^{2}}=\frac{9 \zeta(3) k_{B}^{3} k_{F}}{\pi \hbar v_{F} d \sqrt{\Delta_{M} \Delta_{D}}} .
$$

Here $v_{F}$ and $k_{F}$ are the Fermi velocity and momentum along the nodal directions, respectively; $d$ is the average interlayer distance; $\zeta(3)=1.20$. One should note that impurity scattering tends to suppress the values of both $\frac{\kappa_{e l}}{T}$ and $\frac{C_{e l}}{T^{2}}$. The Fermi velocity along the nodal directions has recently been obtained for YBCO from the studies of surface Andreev bound states [36. The deduced Fermi velocity $v_{F}$ is $(1.2 \pm 0.2) \times 10^{5} \mathrm{~m} / \mathrm{s}$, which is a factor of 2 smaller than the measured Fermi velocity along the diagonal directions from the ARPES data of BSCCO [37. This suggests that the nodal directions might be far away from the diagonal directions. For the $s^{*}$-wave gap function deduced above for overdoped YBCO, the nodal directions are about $19^{\circ}$ away from the diagonal directions (i.e., at $\theta=26^{\circ}$ ). Indeed, from the ARPES data of BSCCO 37, one can clearly see that the Fermi velocity at $\theta=26^{\circ}$ is smaller than that at $\theta=45^{\circ}$ by a factor of about 2. Substituting $v_{F}=1.2 \times 10^{5} \mathrm{~m} / \mathrm{s}, k_{F}$ $=0.7 \AA^{-1} 37, d=5.85 \AA, \Delta_{M}=30 \mathrm{meV}$ and $\Delta_{D}$ $=19 \mathrm{meV}$ into Eq. 7 and Eq. 8, we obtain $\frac{\kappa_{e l}}{T}=0.12$ $\mathrm{mW} / \mathrm{K}^{2} \mathrm{~cm}$ and $\frac{C_{e l}}{T^{2}}=0.24 \mathrm{~mJ} / \mathrm{moleK}^{3}$. The calculated values are in excellent agreement with the measured values: $\frac{\kappa_{e l}}{T}=0.14 \pm 0.03 \mathrm{~mW} / \mathrm{K}^{2} \mathrm{~cm}$ (Ref. 35) and $\frac{C_{e l}}{T^{2}}=$ $0.20 \pm 0.05 \mathrm{~mJ} / \mathrm{moleK}^{3}$ (Ref. [38]).

Moreover, thermal conductivity of $\mathrm{YBCO}$ as a function of angle of an inplane magnetic field relative to the crystal axes has been studied both theoretically and experimentally 39,40. A theoretical calculation for the angular dependence of the magnetothermal conductivity [39] shows that an extended $s$-wave gap produces a more symmetric angular variation than a $d$-wave gap. It 
appears that both sets of experimental data 39.40 are more consistent with an extended $s$-wave gap than a $d$ wave gap.

\section{The pairing symmetry in $\mathrm{La}_{2-x} \mathrm{Sr}_{x} \mathrm{CuO}_{4}$}

The polarized Raman scattering data [41] for nearly optimally-doped $\mathrm{La}_{2-x} \mathrm{Sr}_{x} \mathrm{CuO}_{4}$ (LSCO) with $T_{c}=37$ $\mathrm{K}$ yield $2 \Delta_{M} / k_{B} T_{c}=7.7$. From the measured value of $d \lambda_{a b}(T) /\left[\lambda_{a b}(0) d T\right]$ for the optimally-doped LSCO [42], one can readily calculate $2 \sqrt{\Delta_{M} \Delta_{D}} / k_{B} T_{c}=4.2$ using Eq. 4. Then we get $2 \Delta_{D} / k_{B} T_{c}=2.3$, i.e., $\Delta_{D}=3.8$ meV. This value is in good agreement with the Andreev reflection spectrum of optimally-doped LSCO 43], which shows the $s$-wave like gap feature at $e V \simeq 3.5 \mathrm{meV}$. Therefore, three independent bulk-sensitive experiments on the optimally-doped LSCO consistently suggest a gap function: $\Delta(\theta)=8.1(\cos 4 \theta+0.53) \mathrm{meV}$ with $\Delta_{D}=3.8$ $\mathrm{meV}$ and $\Delta_{M}=12.5 \mathrm{meV}$.

Now we can quantitatively explain the neutron scattering experiment on an optimally-doped LSCO single crystal 44]. The experiment shows that low energy magnetic excitations are peaked at the quartet of wavevectors $(0.5 \pm 0.135,0.5)$ and $(0.5,0.5 \pm 0.135)$ in the normal state, and a spin gap with energy of about $6.7 \mathrm{meV}$ appears in the low-temperature superconducting state. The magnitude of the spin gap should be equal to twice the superconducting gap along the incommensurate wave vectors (i.e., at $\theta=39^{\circ}$ ) 45 . From the gap function deduced above, we calculate $2 \Delta\left(39^{\circ}\right)=6.2 \mathrm{meV}$, in remarkably good agreement with experiment. Moreover, it was also found 44] that the spin gap at $\theta=45^{\circ}$ is $6 \pm 2 \mathrm{meV}$, which is consistent with $2 \Delta_{D}=7.6 \mathrm{meV}$ within the experimental uncertainty. Obviously, the $d$-wave gap function is incompatible with the large spin gap observed along the diagonal direction. The neutron data might be also consistent with an isotropic spin gap, as suggested by Lake et al. 44. However, the isotropic spin gap is incompatible with the $T^{3}$ dependence of the spin-lattice relaxation rate observed in hole-doped cuprates. Only with the $s^{*}$ wave gap function for LSCO, one can quantitatively explain the neutron experiment, Raman scattering, magnetic penetration depth, Andreev reflection, and magnetic resonances.

\section{THE PAIRING SYMMETRY IN ELECTRON-DOPED CUPRATES}

The recent measurements of $\lambda_{a b}(T)$ in an electrondoped $\operatorname{Pr}_{1.85} \mathrm{Ce}_{0.15} \mathrm{CuO}_{4-y}$ (PCCO) reveal contradictory results 46, 47. In a high-quality PCCO thin film with the lowest residual resistivity and the highest $T_{c}$, the temperature dependence of $\left[\lambda_{a b}(T)-\lambda_{a b}(0)\right] / \lambda_{a b}(0)$ is consistent with an $s$-wave pairing symmetry with a reduced energy gap $2 \Delta(0) / k_{B} T_{c}=2.946$. On the other hand, the lowtemperature $\lambda_{a b}(T)$ in less ideal PCCO single crystals exhibits a power-law temperature dependence, as expected from a dirty $d$-wave superconductor 47 .

We show that these apparently conflicting data might well be reconciled by a deeper understanding of how microstructure affects screening. It is well known, for example, that the screening length in weakly coupled Josephson array of grains is dominated by the magnitude and temperature dependence of the Josephson coupling current between array elements [48]. Thus, tunnel coupling across grain boundaries and/or planar defects (weak links), rather than the BCS response of the grains themselves, mainly determines the magnetic screening length, surface resistance, and critical current (see a review article [49]). The extrinsic effect due to the weak links can lead to a linear $T$ dependence in the effective $\lambda_{a b}(T)$ at low temperatures and to a large residual surface resistance [50]. Similarly, Hebard et al. [51] showed that the current-induced nucleation of vortex-antivortex pairs at defects can make an additional extrinsic contribution to the screening length, i.e., a pinning penetration depth $\lambda_{a b}^{p}(T)$. Within this scenario, the $\lambda_{a b}^{p}(T)$ in zero magnetic field is given by 51 .

$$
\lambda_{a b}^{p}(t)=\lambda_{a b}^{p}(0) /\left(1-t^{2}\right),
$$

where $t=T / T_{c} ; \lambda_{a b}^{p}(0)=\left[\Phi_{0} / H_{c}(0)\right] \sqrt{2 N_{d} / \pi} ; \Phi_{0}$ is the flux quantum; $N_{d}$ is the areal density of uniformly distributed defects; $H_{c}(0)$ is the zero-temperature critical field. In the presence of the external dc field $H$, the expression for $\lambda_{a b}^{p}(0, H)$ has to be modified [50]. The total screening length is $\lambda_{a b}(t)=\sqrt{\left[\lambda_{a b}^{L}(t)\right]^{2}+\left[\lambda_{a b}^{p}(t)\right]^{2}}$, where $\lambda_{a b}^{L}(t)$ is the intrinsic London penetration depth [51]. Assuming an $s$-wave pairing symmetry, we readily show that the $\lambda_{a b}(T)$ at low temperatures (below $0.2 \Delta(0) / k_{B}$ ) is given by

$$
\begin{gathered}
\lambda_{a b}(T)=\lambda_{a b}(0)+\frac{\left[\lambda_{a b}^{L}(0)\right]^{2}}{\lambda_{a b}(0)} \sqrt{\pi \Delta(0) / 2 k_{B} T} \\
\exp \left[-\Delta(0) / k_{B} T\right]+\frac{\lambda_{a b}^{2}(0)-\left[\lambda_{a b}^{L}(0)\right]^{2}}{\lambda_{a b}(0) T_{c}^{2}} T^{2} .
\end{gathered}
$$

It is clear that the $T^{2}$ dependence of $\lambda_{a b}(T)$ at low temperatures in zero field can be completely caused by the extrinsic effect, that is, the nucleation of vortexantivortex pairs at defects. If $N_{d}$ is negligible, $\lambda_{a b}(0)=$ $\lambda_{a b}^{L}(0)$, and the second term in Eq. 10 is absent. Then we recover the BCS expression [52],

$$
\lambda_{a b}(T)=\lambda_{a b}(0)+\lambda_{a b}(0) \sqrt{\pi \Delta(0) / 2 k_{B} T} \exp \left[-\Delta(0) / k_{B} T\right] .
$$

In Fig. 7a, we plot temperature dependence of $\lambda_{a b}(T)$ below $6 \mathrm{~K}$ for a PCCO single crystal (the data are from 
Ref. 47). The zero-temperature in-plane penetration depth $\lambda_{a b}(0)$ was measured to be $2500 \AA$ 47. This crystal shows $T_{c}^{\text {onset }}$ at $22 \mathrm{~K}$ (defined by the onset of diamagnetism), and $T_{c}^{\text {mid }}$ at $19 \mathrm{~K}$ (defined as the inflection point on $\lambda_{a b}(T)$ ) [47]. A wide superconducting transition in this crystal manifests a rather low quality of the crystal.

We fit the data by Eq. 10 with two fitting parameters $\Delta(0)$ and $\lambda_{a b}^{L}(0)$, and with a fixed $T_{c}=20.5 \mathrm{~K}$ (the average of $T_{c}^{o n s e t}$ and $\left.T_{c}^{\text {mid }}\right)$. The solid line is the fitted curve by Eq. 10. It is remarkable that the fit is very good. This can be seen more clearly in Fig. 7b where the difference between the data and the fitted curve is plotted. There is a negligible systematic error (the deviation is less than the magnitude of the data scattering). From the fit, we find $\Delta(0) / k_{B}=29.6 \pm 0.1 \mathrm{~K}$, and $\lambda_{a b}^{L}(0)=1643 \AA$. The deduced $\lambda_{a b}^{L}(0)$ is in excellent agreement with the value (1600 $\pm 100 \AA)$ obtained from the optical data [53]. The magnitude of $2 \Delta(0) / k_{B} T_{c}=2.9$ is also the same as the one deduced from a high-quality film where the $T^{2}$ term is absent 46. The value of $\Delta(0)$ justifies the fit to the data below $6 \mathrm{~K}$, namely, $0.2 \Delta(0) / k_{B}$. Therefore, the $\lambda_{a b}(T)$ data for the crystal are in quantitative agreement with an anisotropic $s$-wave pairing symmetry with no nodes.

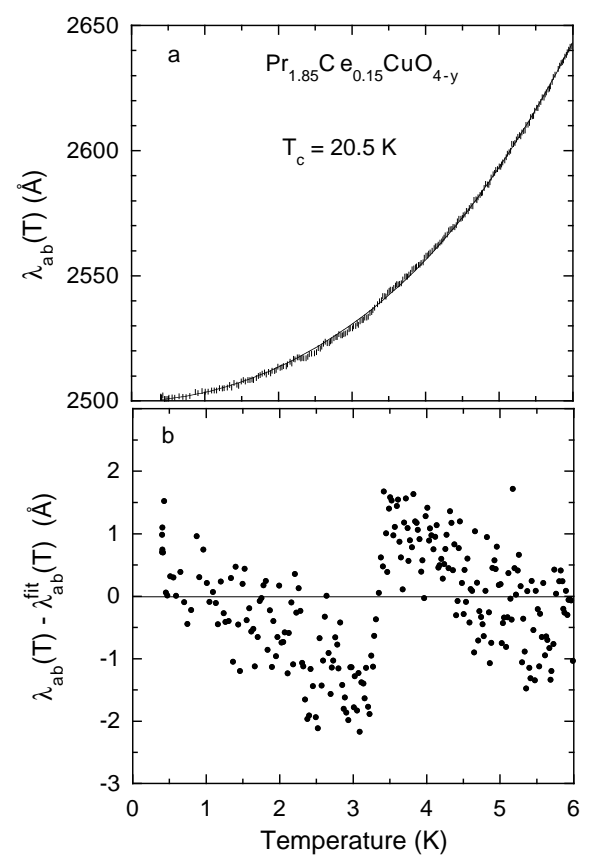

FIG. 7. (a) Temperature dependence of $\lambda_{a b}(T)$ below $6 \mathrm{~K}$ for a PCCO single crystal. The solid line is the fitted curve by Eq. 10 with $2 \Delta(0) / k_{B} T_{c}=2.9$ and $\lambda_{a b}^{L}(0)=1643 \AA$. The value of $\lambda_{a b}^{L}(0)$ was found to be $1600 \pm 100 \AA$ from the optical data [53]. (b) The difference between the data and the fitted curve. The data are from Ref. 447.

From the values of $\lambda_{a b}^{L}(0)$ and $\lambda_{a b}(0)$, we calcu- late $\lambda_{a b}^{p}(0)=1884 \AA$. Using the relation $\lambda_{a b}^{p}(0)=$ $\left[\Phi_{0} / H_{c}(0)\right] \sqrt{2 N_{d} / \pi}$ and $H_{c}(0)=2 \mathrm{kOe}$ [54, we estimate $N_{d}=5.2 \times 10^{10} / \mathrm{cm}^{2}$, corresponding to one defect over $1333 \mathrm{Cu}$ sites. This implies that a small density of defects can produce a quite large $\lambda_{a b}^{p}(0)$ which contributes a substantial $T^{2}$ term in $\lambda_{a b}(T)$.

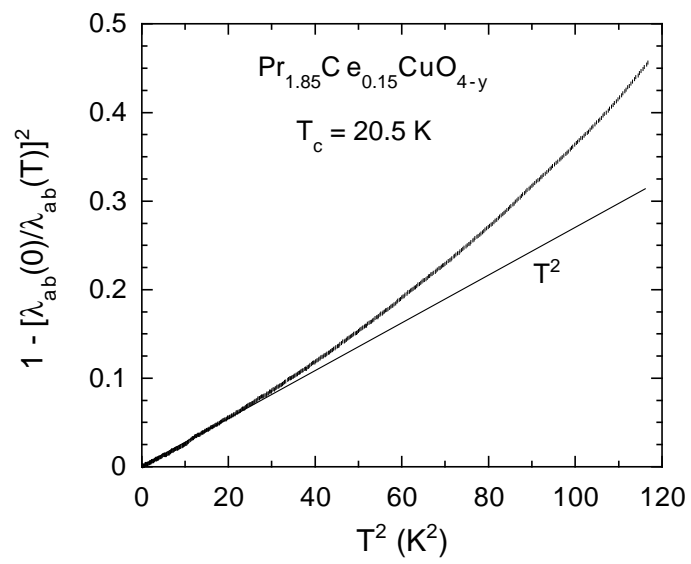

FIG. 8. The $T^{2}$ dependence of the quantity $1-\lambda_{a b}^{2}(0) / \lambda_{a b}^{2}(T)$ over $0.4-10.8 \mathrm{~K}$ for the same PCCO crystal as the one in Fig. 17. The crossover from the $T^{2}$ to a higher power-law dependence starts at about $5 \mathrm{~K}$. There is no crossover from the $T^{2}$ to the $\mathrm{T}$ dependence at $T^{*}$ $\simeq 9 \mathrm{~K}$.

In order to rule out the possibility that the data can be also consistent with a $d$-wave symmetry in the dirty limit, we plot the data as $1-\lambda_{a b}^{2}(0) / \lambda_{a b}^{2}(T)$ vs $T^{2}$ in Fig. 8. It is apparent that the quantity $1-$ $\lambda_{a b}^{2}(0) / \lambda_{a b}^{2}(T)$ is proportional to $T^{2}$ below about $5 \mathrm{~K}$. For a dirty $d$-wave superconductor, a crossover from $T^{2}$ to $T$ dependence should be seen at a temperature $T^{*} \simeq \lambda_{a b}(0) \ln 2 /\left[\Delta_{M}(0) d \lambda_{a b} / d T^{2}\right]$, where $\Delta_{M}(0)$ is the maximum gap at zero temperature [55]. Using $\lambda_{a b}(0)$ $=2500 \AA$ [4], $d \lambda_{a b} / d T^{2}=3.7 \AA / \mathrm{K}^{2}$ 47], and $\Delta_{M}(0)$ $=2.5 T_{c}$ 56, one finds $T^{*} \simeq 9 \mathrm{~K}$. There is no such a crossover at any temperatures up to $11 \mathrm{~K}$ (see Fig. 8). Only a possible crossover from the $T^{2}$ to a higher powerlaw dependence is seen at about $5 \mathrm{~K}$. Therefore, the data cannot agree with the $d$-wave pairing symmetry. Furthermore, the absence of the linear $T$ term in $\lambda_{a b}(T)$ indicates that the extrinsic contribution to $\lambda_{a b}(T)$ due to weak links [50] is negligible in this crystal.

In Fig. 9, we show $\left[\lambda_{a b}(T)-\lambda_{a b}(0)\right] / \lambda_{a b}(0)$ as a function of temperature for a high-quality PCCO thin film (the data are from Ref. 46). The film has the lowest residual resistivity $(<50 \mu \Omega \mathrm{cm})$ and the highest $T_{c}(24$ $\mathrm{K})$ reported for the $\mathrm{PCCO}$ system [46]. This indicates a high-quality of the film, which was grown using molecular beam epitaxy. The optimal quality of the film may be due to the fact that a homogeneous oxygen reduction can be easily achieved in thin films. Since the data at low temperatures are quite flat, it appears that there is 
neither $T^{2}$ nor $T$ contribution. We thus fit the data below $6.5 \mathrm{~K}$ by Eq. 11 with one fitting parameter $\Delta(0)$. The best bit gives $\Delta(0) / k_{B}=31.9 \pm 0.1 \mathrm{~K}$, which justifies the fit to the data below $6.5 \mathrm{~K}\left(\sim 0.2 \Delta(0) / k_{B}\right)$. This leads to $2 \Delta(0) / k_{B} T_{c}=2.7$, which is nearly the same as that deduced above for the less ideal crystal where there is a significant $T^{2}$ term in $\lambda_{a b}(T)$ due to the existence of defects. All these results consistently suggest that the pairing symmetry in electron-doped cuprates is the anisotropic $s$-wave with no line nodes.

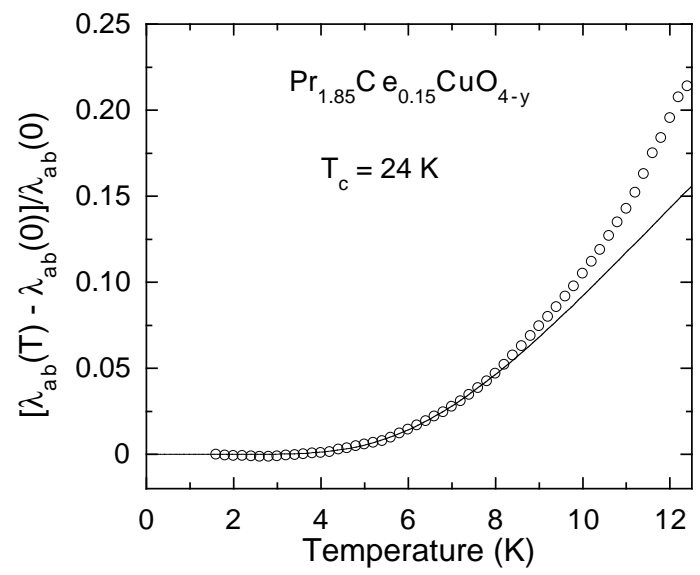

FIG. $\quad 9$. Temperature

dependence

of $\left[\lambda_{a b}(T)-\lambda_{a b}(0)\right] / \lambda_{a b}(0)$ for a high-quality PCCO thin film with the lowest residual resistivity and the highest $T_{c}$. The solid line is the fitted curve by Eq. 11 with $2 \Delta(0) / k_{B} T_{c}=$ 2.7. The data are from Ref. 46].

Polarized Raman scattering [56 has also shown that the symmetry of the order parameter in $\mathrm{Nd}_{1.84} \mathrm{Ce}_{0.16} \mathrm{CuO}_{4-y}$ (NCCO) is consistent with an anisotropic $s$-wave. More precisely, the tunneling spectra [57] are consistent with a gap function: $\Delta(\theta)=$ $\Delta(s+\cos 4 \theta)$ with $s>1$. If we use $\Delta_{M}=2.5 T_{c}$ [56] and the minimum gap $\Delta_{m}=1.4 T_{c}$ (from the $\lambda_{a b}(T)$ data), we find $\Delta(\theta)=1.15(3.52+\cos 4 \theta) \mathrm{meV}$ for an electron-doped cuprate with $T_{c}=24 \mathrm{~K}$. Therefore, three bulk-sensitive experiments consistently indicate an anisotropic $s$-wave pairing symmetry in electron-doped cuprates.

\section{PHASE-SENSITIVE EXPERIMENTS ALONG THE C-AXIS DIRECTION}

The most reliable phase-sensitive experiment is the atomically clean BSCCO Josephson junctions between identical single crystal cleaves stacked and twisted an angle $\phi_{0}$ about the c-axis [12]. The quality of the junction is nearly the same as that of the intrinsic Josephson junctions made of the Bi-O insulating layers. Theoretically, it has been shown that the critical current $I_{c}$ of the twist junction is 58

$$
I_{c} \propto \sum_{\ell} \eta_{\ell} \Delta_{l} \cos \ell \phi_{0}
$$

where $\ell=0,1,2, \ldots$, and $\eta_{\ell}<<\eta_{0}$ for $\ell \geq 1$. The above equation indicates that the $s$-wave component contributes to the critical current much more effectively. The experiment shows 12] that the $I_{c}$ value is nearly independent of the twist angle $\phi_{0}$, and the temperature dependence of $I_{c}$ is consistent with the Ambegaokar-Baratoff (AB) model for an $s$-wave superconductor. This indicates that the $s$-wave component in this material must be significant compared with the other high angular momentum components. For slightly overdoped BSCCO, we have found that the gap function is $\Delta(\theta)=17.75(\cos 4 \theta+$ $0.46) \mathrm{meV}$ for $T_{c}=90 \mathrm{~K}$, and $\Delta(\theta)=18(\cos 4 \theta+0.33)$ $\mathrm{meV}$ for $T_{c}=86 \mathrm{~K}$. Then we have $\Delta_{s}=6-8 \mathrm{meV}$, which is not small compared with the g-wave component $\Delta_{g}=18$ meV. Since $\eta_{4}<<\eta_{0}$ [58], the dominant contribution to the $I_{c}$ should be the $s$-wave component, as observed [12]. From the magnitude of the $s$-wave component, we can calculate $I_{c} R_{N}=(\pi / 2 e) \Delta_{s}=9-12 \mathrm{mV}$. The measured $I_{c} R_{N}$ value is about $8 \mathrm{mV}$ [12. This is in quantitative agreement with the predicted value considering the fact that the strong coupling effect can reduce the $I_{c} R_{N}$ value by more than $20 \%$.

Another reliable phase-sensitive experiment is the caxis $\mathrm{Pb} / \mathrm{YBa}_{2} \mathrm{Cu}_{3} \mathrm{O}_{7-y}$ Josephson junction experiment [13. This junction can be described as SINS' (where S $=\mathrm{YBCO}, \mathrm{S}^{\prime}=\mathrm{Pb}, \mathrm{I}$ and $\mathrm{N}$ represent the insulating and normal-metal layers, respectively). Due to a very short coherent length $\xi_{c}$ along the c-axis direction, the bulk gap will be strongly depressed at the SI interface; the depression factor is $c / \xi_{c}$ (where $c$ is the lattice constant along the c-axis) 59]. From $\xi_{c}=\xi_{a b} / \gamma$ (where $\gamma$ is the mass anisotropy parameter and equal to about 8 for optimallydoped YBCO 60]), we get $\xi_{c}=1.7 \AA$ by taking $\xi_{a b}=$ $14 \AA$. Therefore, the gap size at SI interface will be suppressed by a factor of about 7 . Since the bulk $s$-wave component $\Delta_{s}$ in slightly overdoped YBCO is $3-5 \mathrm{meV}$ (see above), this component at the SI interface should be reduced to $0.4-0.7 \mathrm{meV}$. Then the $I_{c} R_{N}$ value is calculated to be $0.93-1.27 \mathrm{mV}$, in quantitative agreement with the measured one $(\sim 0.9 \mathrm{mV})[13]$.

Now we discuss another c-axis Josephson tunneling experiments in which a conventional superconductor $(\mathrm{Pb})$ is deposited across a single twin boundary of a YBCO crystal 61]. Because $\mathrm{Pb}$ is an $s$-wave superconductor, the $\mathrm{Pb}$ counterelectrode couples only to the $s$-wave component of the YBCO order parameter. If YBCO were predominantly $d$-wave, any small $s$-wave component added to the dominant $d$-wave component would change sign across the twin boundary. In this case, magnetic fields parallel to the boundary would produce a local minimum in $I_{c}$ at $B=0$, in agreement with the observation 61]. The experimental results thus appear to provide evidence for mixed $d$ - and $s$-wave pairing symmetry in the bulk with 
a reversal in the sign of the $s$-wave component across the boundary. However, if the bulk OP symmetry in a single domain were $d+s$ or $d-s$, one would expect a nearly zero $I_{c}$ in heavily twinned crystals. The fact that the observed $I_{c} R_{N}$ in heavily twinned crystals 13 is nearly the same as the one in the single-domain crystal [61] rules out the bulk $d+s$ or $d-s$ wave OP symmetry in YBCO. Therefore the only possibility is that a half/fractional flux is trapped in the twin boundary. Also, this can naturally explain why $I_{c}$ does not go to zero even for a symmetric junction with the same junction area in both sides of the twin boundary 61.

\section{PHASE-SENSITIVE EXPERIMENTS ALONG THE AB-PLANES}

The phase-sensitive tricrystal experiments on both hole and electron-doped cuprates [20,9, 10] show that the OP symmetry is the $d$-wave, in contradiction with the above conclusion drawn from many bulk-sensitive experiments. In order to resolve the above discrepancy, one should notice that the tricrystal experiments are rather surface sensitive, so these experiments are probing the OP symmetry at the surface/interface, rather than in the bulk. Based on the Ginzburg-Landau free energy, Bahcall [18] has shown that the OP symmetry near surfaces/interfaces can be different from that in the bulk if the bulk OP is strongly suppressed at the surfaces. Experimentally, the observed $I_{c} R_{N}$ values in all the tricrystal experiments are about two order of magnitude smaller than the intrinsic Ambegaokar-Baratoff limit. For example, in the optimally-doped $\mathrm{YBa}_{2} \mathrm{Cu}_{3} \mathrm{O}_{7-y}$, the magnitude of the maximum gap $\Delta_{M}(0)$ is about $30 \mathrm{meV}$ 8, 17. Then the intrinsic $I_{c} R_{N}$ value should be equal to the Ambegaokar-Baratoff limit $\pi \Delta_{M}(0) / 2 e=47 \mathrm{mV}$, which has been confirmed by a nearly ideal SIS break junction experiment [17]. However, the observed $I_{c} R_{N}$ values in the tricrystal experiments on $\mathrm{YBa}_{2} \mathrm{Cu}_{3} \mathrm{O}_{7-y}$ and $\mathrm{Tl}_{2} \mathrm{Ba}_{2} \mathrm{CuO}_{6+y}$ [9, 10 are about $1.8 \mathrm{mV}$ and $0.5 \mathrm{mV}$, respectively. These values are about two order of magnitude smaller than the intrinsic bulk values. Similarly, the observed $I_{c} R_{N}$ value in the NCCO and PCCO tricrystal experiments is about $0.1 \mathrm{mV}$, as inferred from the measured critical current density $J_{c}=6 \mathrm{~A} / \mathrm{cm}^{2}$ 20] and the empirical relation between $I_{c} R_{N}$ and $J_{c}$ 62. This $I_{c} R_{N}$ value is also about two order of magnitude smaller than the intrinsic bulk value, which is estimated to be $\sim 8 \mathrm{mV}$ with $\Delta_{M}(0)=2.5 T_{c}$ [56]. Therefore, the OP at the interfaces of the grain boundary junctions must be strongly depressed in order to explain such small $I_{c} R_{N}$ values. This strong depression in the order parameter ensures the condition under which the OP symmetry near surfaces/interfaces can be different from that in the bulk [18]. Hence, it is very likely that the tricrystal experiments are detecting the OP symmetry at the degraded interfaces, which may be different from the intrinsic one in the bulk.

Now the question arises: why the bulk OP can be so strongly depressed at the surfaces of the grain boundary junctions? It is known that the coherent length in cuprates is very short due to a large superconducting gap and small Fermi velocity. The short coherent length in cuprates can lead to a large depression of the OP near the interfaces even within the conventional theory of the proximity effect [59,63. Alternatively, several groups 64 66 showed that there are possibly nonsuperconducting regions near the boundary of the junction due to hole depletion and/or strain, so that the critical current density can be reduced by several order of magnitude compared with the intrinsic bulk value.

There is another way to explain the tricrystal experiments. As discussed above, the bounaries of the grainboundary junctions are intrinsically underdoped superconductors or nonsuperconducting antiferromagnets due to hole depletion and/or strain 64 67]. For underdoped cuprates, the superconductivity mainly arises from the Bose-Einstein condensation of preformed pairs [68]. In this case, the symmetry of the superconducting condensate is different from the pairing symmetry; the former is the $d$-wave while the latter might be $s$-wave [68]. Since Josephson tunneling probes the symmetry of the superconducting condensate, the $d$-wave symmetry of the condensate is consistent with the tricrystal experiments.

\section{CONCLUSION}

In conclusion, the existing bulk and nearly bulksensitive experiments consistently show that the dominant bulk pairing symmetry in hole-doped cuprates is of extended $s$-wave with eight line nodes, and of anisotropic $s$-wave in electron-doped cuprates. The deduced extended $s$-wave pairing symmetry for hole-doped cuprates is also in quantitative agreement with the phase-sensitive experiments along the $\mathrm{c}$-axis for both $\mathrm{Bi}_{2} \mathrm{Sr}_{2} \mathrm{CaCu}_{2} \mathrm{O}_{8+y}$ and $\mathrm{YBa}_{2} \mathrm{Cu}_{3} \mathrm{O}_{7-y}$. The proposed pairing symmetries do not contradict some surface- and phase-sensitive experiments which show a predominant $d$-wave pairing symmetry at the degraded surfaces.

Acknowlegement: The author would like to thank R. Prozorov, L. Alff, S. Kamal and W. N. Hardy for sending their published data. 
[1] W. N. Hardy, D. A. Bonn, D. C. Morgan, Ruixing Liang, and K. Zhang, Phys. Rev. Lett. 70, 3999 (1993).

[2] T. Jacobs, S. Sridhar, Q. Li, G. D. Gu, and N. Koshizuka, Phys. Rev. Lett. 75, 4516 (1995).

[3] S.-F. Lee, D. C. Morgan, R. J. Ormeno, D. Broun, R. A. Doyle, J. R. Waldram, and K. Kadowaki, Phys. Rev. Lett. 77, 735 (1996).

[4] A. Bhattacharya, I. Zutic, O. T. Valls, A. M. Goldman, U. Welp, and B. Veal, Phys. Rev. Lett. 82, 3132 (1999).

[5] M. Willemin, C. Rossel, J. Hofer, H. Keller, Z. F. Ren, and J. H. Wang, Phys. Rev. B 57, 6137 (1998).

[6] A. Sacuto, R. Combescot, N. Bontemps, P. Monod, V. Viallet, and D. Colson, Europhys. Lett. 39, 207 (1997).

[7] C. Kendziora, R. J. Kelley, and M. Onellion, Phys. Rev. Lett. 77, 727 (1996).

[8] J.Y.T. Wei, N.-C. Yeh, D. F. Garrigus, and M. Strasik, Phys. Rev. Lett. 81, 2542 (1998).

[9] C. C. Tsuei, J. R. Kirtley, M. Rupp, J. Z. Sun, L.-S. Yu-Jahnes, C. C. Chi, A. Gupta, and M. B. Ketchen, J. Phys. Chem. Solids, 56, 1787 (1995).

[10] C. C. Tsuei, J. R. Kirtley, M. Rupp, J. Z. Sun, A. Gupta, M. B. Ketchen, C. A. Wang, Z. F. Ren, J. H. Wang, and M. Bhushan, Science, 271, 329 (1996).

[11] J. R. Kirtley, C. C. Tsuei, and K. A. Moler, Science, 285, 1373 (1999).

[12] Q. Li, Y. N. Tsay, M. Suenaga, R. A. Klemm, G. D. Gu, and N. Koshizuka, Phys. Rev. Lett. 83, 4160 (1999).

[13] A. G. Sun, D. A. Gajewski, M. B. Maple, and R. C. Dynes, Phys. Rev. Lett. 72, 2267 (1994).

[14] H. Ding, J. C. Campuzano, A. F. Bellman, T. Yokoya, M. R. Norman, M. Randeria, T. Takahashi, H. KatayamaYoshida, T. Mochiku, K. Kadowaki, and G. Jennings, Phys. Rev. Lett. 74, 2784 (1995).

[15] R. J. Kelley, C. Quitmann, M. Onellion, H. Berger, P. Almeras, and G. Margaritondo, Science 271, 1255 (1996).

[16] I. Vobornik, R. Gatt, T. Schmauder, B. Frazer, R. J. Kelley, C. Kendziora, M. Grioni, M. Onellion, and G. Margaritondo, Physica C 317-318, 589 (1999).

[17] Ya. G. Ponomarev et al., Physica C 243, 167 (1995).

[18] S. R. Bahcall, Phys. Rev. Lett. 76, 3634 (1996).

[19] J. Ma, C. Quitmann, R. J. Kelley, G. Margaritondo, and M. Onellion, Solid State Commun. 94, 27 (1995).

[20] C. C. Tsuei and J. R. Kirtley, Phys. Rev. Lett. 85, 182 (2000).

[21] N. Miyakawa, P. Guptasarma, J. F. Zasadinski, D. G. Hinks, and K. E. Gray, Phys. Rev. Lett. 80, 157 (1998).

[22] Y. DeWilde, N. Miyakawa, P. Guptasarma, M. Iavarone, L. Ozyuzer, J. F. Zasadinski, P. Romano, D. G. Hinks, C. Kendziora, C. W. Crabtree, and K. E. Gray, Phys. Rev. Lett. 80, 153 (1998).

[23] I. Kosztin and A. J. Leggett, Phys. Rev. Lett. 79, 135 (1997).

[24] K. Suzuki, K. Ichimura, K. Nomura, and S. Takekawa, Phys. Rev. Lett. 83, 616 (1999).

[25] A. Mourachkine, cond-mat/9901282.

[26] V. M. Krasnov, A. Yurgens, D. Winkler, P. Delsing, and T. Claeson, Phys. Rev. Lett. 84, 5860 (2000).

[27] R. Prozorov, R. W. Giannetta, A. Carrington, P. Fournier, R. L. Greene, P. Guptasarma, D. G. Hinks, and A. R. Banks, Appl. Phys. Lett. 77, 4202 (2000).
[28] L. Buschmann, M. Boekholt, and G. Güntherodt, Physica C 203, 68 (1992).

[29] L. Ozyuzer, J. F. Zasadzinski, C. Kendziora, and K. E. Gray, Phys. Rev. B 61, 3629 (2000).

[30] I. Maggio-Aprile, Ch. Renner, A. Erb, E. Walker, and O. Fisher, Phys. Rev. Lett. 75, 2754 (1995).

[31] Y. Yagil, N. Hass, G. Desgardin, and I. Monot, Physica C 250, 59 (1995).

[32] S. Kamal, R.X.Liang, A. Hosseini, D. A. Bonn, and W. N. Hardy, Phys. Rev. B 58, R8933 (1998).

[33] D. Thelen, D. Pines, and J. P. Liu, Phys. Rev. B 47, 9151 (1993).

[34] I. Zutic and O. T. Valls, Phys. Rev. B 56, 11279 (1997).

[35] M. Chiao, R. W. Hill, C. Lupien, L. Taillefer, P. Lambert, R. Gagnon, and P. Fournier, Phys. Rev. B 62, 3554 (2000).

[36] A. Carrington, F. Manzano, R. Prozorov, R. W. Giannetta, N. Kameda, and T. Tamegai, Phys. Rev. Lett. 86, 1074 (2001).

[37] A. Kaminski, M. Randeria,J. C. Campuzano, M. R. Norman, H. Fretwell, J. Mesot, T. Sato, T. Takahashi, and K. Kadowaki, Phys. Rev. Lett. 86, 1070 (2001).

[38] A. Junod, B. Revaz, Y. Wang, and A. Erb, Physica B 284-288, 1043 (2000).

[39] F. Yu, M. B. Salamon, A. J. Leggett, W. C. Lee, and D. M. Ginsberg, Phys. Rev. Lett. 74, 5136 (1995).

[40] H. Aubin, K. Behnia, M. Ribault, R. Gagnon, and L. Taillefer, Phys. Rev. Lett. 78, 2624 (1997).

[41] X. K. Chen, J. C. Irwin, H. J. Trodahl, T. Kimura, and K. Kishio, Phys. Rev. Lett. 73, 3290 (1994).

[42] L. Panagopoulos et al., Phys. Rev. B 60, 14617 (1999).

[43] G. Deutscher, N. Achsaf, D. Goldschmidt, A. Revcolevschi, and A. Vietkine, Physica C 282-287, 140 (1997).

[44] B. Lake, G. Aeppli, T. E. Mason, A. Schröder, D. F. McMorrow, K. Lefmann, M. Isshiki, M. Nohara, H. Takagi, and S. M. Hayden, Nature (London). 400, 43 (1999).

[45] T. E. Mason, A. Schröder, G. Aeppli, H. A. Mook, and S. M. Hayden, Phys. Rev. Lett. 77, 1604 (1996).

[46] L. Alff, S. Meyer, S. Kleefisch, U. Schoop, A. Marx, H. Sato, M. Naito, and R. Gross, Phys. Rev. Lett. 83, 2644 (1999).

[47] R. Prozorov, R. W. Giannetta, P. Fournier, and R. L. Greene, Phys. Rev. Lett. 85, 3700 (2000).

[48] B. Giovannini and L. Weiss, Solid State Commun. 27, 1005 (1978).

[49] J. Halbritter, Supercond. Sci. Technol. 12, 883 (1999).

[50] J. Halbritter, J. Appl. Phys. 71, 339 (1992).

[51] A. F. Hebard, A. T. Fiory, M. P. Siegal, J. M. Phillips, and R. C. Haddon, Phys. Rev. B 44, 9753 (1991).

[52] B. Mühlschlegel, Z. Phys. 155, 313 (1959).

[53] C. C. Homes, B. P. Clayman, J. L. Peng, and R. L. Greene, Phys. Rev. B 56, 5525 (1997).

[54] D. H. Wu, J. Mao, S. N. Mao, J. L. Peng, X. X. Xi, T. Venkatesan, and R. L. Greene, Phys. Rev. Lett. 70, 85 (1993).

[55] P. J. Hirschfeld and N. Goldenfeld, Phys. Rev. B 48, 4219 (1993).

[56] B. Stadlober, G. Krug, R. Nemetschek, R. Hackl, J. L. 
Cobb, and J. T. Markert, Phys. Rev. Lett. 74, 4911 (1995).

[57] S. Kashiwaya, T. Ito, K. Oka, S. Ueno, H. Takashima, M. Koyanagi, Y. Tanaka, and K. Kajimura, Phys. Rev. B 57, 8680 (1998).

[58] R. A. Klemm, C. T. Rieck, and K. Scharnberg, Phys. Rev. B 58, 1051 (1998).

[59] K. A. Müller, Nature (London) 377, 133 (1995); G. Deutscher and K. A. Müller, Phys. Rev. Lett. 59, 1745 (1987).

[60] M. Willemin, A. Schilling, H. Keller, C. Rossel, J. Hofer, U. Welp, W. K. Kwok, R. J. Olsson, and G. W. Crabtree , Phys. Rev. Lett. 81, 4236 (1998).

[61] K. A. Kouznetsov et al. , Phys. Rev. Lett. 79, 3050 (1997).

[62] S. Kleefisch, L. Alff, U. Schoop, A. Marx, R. Gross, M. Naito, and H. Sato, Appl. Phys. Lett. 72, 2888 (1998).

[63] M. Y. Kupryanov and K. K. Likharev, IEEE Trans. Magn. 27, 2400 (1991).

[64] J. Halbritter, Phys. Rev. B 46, 14861 (1992).

[65] J. Betouras and R. Joynt, Physica C 250, 256 (1995).

[66] A. Gurevich, E. A. Pashitskii, Phys. Rev. B 57, 13878 (1998).

[67] J. Mannhart and H. Hilgenkamp, Physica C 317-318, 383 (1999).

[68] A. S. Alexandrov, Physica C 305, 46 (1998). 